\title{
L'énergie marémotrice en 1982
}

\section{Tidal energy in 1982}

\author{
M. Banal \\ Président de la Société Hydrotechnique \\ de France
}

Parmi les grands aménagements hydroélectriques projetés dans le monde, les usines marémotrices tiennent une place honorable et originale. Il était donc parfaitement justifié de les évoquer dans le cadre de ces réunions même si aucun de ces grands projets n'a encore fait l'objet d'un début de réalisation. Quinze ans après sa mise en service, l'usine de la Rance reste en effet un exemplaire unique.

Pourtant, elle a rendu exactement les services qu'on attendait d'elle et fournit à elle seule la moitié de l'énergie électrique produite en Bretagne. Dix ans après la session de Novembre 1972 du Comité Technique de la $\mathrm{SHF}^{(1)}$, le bilan favorable dressé à ce moment là pour les six premières années d'exploitation reste entièrement confirmé :

- Production conforme aux prédictions;

- Excellente tenue des ouvrages aux agressions de la mer;

- Acceptation par le milieu.

Pour être complet, il faut cependant signaler, dans les maladies de jeunesse inévitables pour une réalisation originale (on oublie parfois que les groupes de la Rance sont parmi les premiers groupes bulbes de grande purssance mis en service), les défauts constatés en 1975 sur les alternateurs, ce qui a nécessité la réfection successive de tous les groupes.

Il ne s'agit pas d'ailleurs de défauts spécifiques à des groupes marémoteurs, mais accélérés cependant par les démarrages et changements de marche très fréquents. Pendant la période des réparations, 3 a 4 groupes ont été maintenus à l'arrêt, le pompage et le turbinage inversé abandonnés sur les autres. Mais voici quelques informations sur l'usine de la Rance :

- la réparation des alternateurs s'achève et le pompage ainsi que le double effet sont repris progressivement;

(1) Houille Blanche ${ }^{\circ} 2 / 3,1973$.
- le modèle mathématique qui permet de programmer l'exploitation de l'usine, en fonction de la marée et des besoins du réseau, a été réécrit en tenant compte de l'expérience et des progrès de l'informatique ${ }^{(2)}$. A cette occasion, les données introduites dans le modèle ont été vérifiées et une excellente adéquation a été obtenue entre l'exploitation prévue et l'exploitation réelle.

Pour 1981, la production brute a été $570 \mathrm{GWh}$ dont il faut déduire l'énergie de pompage $62 \mathrm{GWh}$ et la consommation des auxiliaires $8 \mathrm{GWh}$, soit une production nette de $500 \mathrm{GWh}$.

Cette valeur est inférieure au productible indiqué dans tous les documents sur l'usine de la Rance.

Y-a-t-il donc eu surestimation de la production?

Il n'en est rien et la différence entre production réelle et production prévue a trois causes :

- d'abord et surtout, la réparation des alternateurs a rendu encore indisponible, en 1981, 3 à 4 groupes (sur 24) en moyenne, entraînant une disponibilité moyenne de $84,5 \%$, alors que le productible avait été évalué avec une disponibilité de $95 \%$, obtenue d'ailleurs pendant les années 73 et 74 avant la constatation des défauts sur les groupes;

- dans l'estimation des $544 \mathrm{GWh}$, la consommation des auxiliaires n'était pas déduite ( $8 \mathrm{GWh}$ environ);

- l'exploitation est conduite, non pas pour maximiser la production, mais pour maximiser la valeur de cette production pondérée en fonction de la valeur de l'énergie à chaque instant. Plus les coûts sont différenciés et plus la production réelle est réduite par rapport à la production maximale théorique. Or, la différence très importante entre les coûts marginaux du kilowattheure nucléaire et du kilowattheure pétrole ou charbon, entraîne des coûts très différenciés.

(2) Bulletin de la Direction des Etudes et Recherches d’E.D.F. Séric B, n 3, 1980.

LA HOUILLE BLANCHE/N ${ }^{\circ}$ 5/6-1982 
A contrario, il faut rappeler que toutes les années ne sont pas identiques du point de vue de la marée, car l'amplitude moyenne varie dans l'intervalle $\pm 3,5 \%$ sur une période de 18 ans 2/3 et l'année 1981 était une année assez forte.

Quand la production réelle est corrigée de tous ces facteurs, on obtient un résultat très proche de $544 \mathrm{GWh}$, prévision faite avant la mise en service de l'usine et qui demeure la meilleure évaluation du productible de l'usine de la Rance.

Mais mon objet est de parler des autres et plus vastes projets marémoteurs. Il n'est peutêtre pas inutile de rappeler d'abord quelques ordres de grandeur de cette ressource énergétique et des principaux sites dans le monde où son utilisation peut être envisagée.

On évalue entre 10 et 15000 TWh l'énergie dissipée annuellement par les marées océaniques (essentiellement dans les mers peu profondes parcourues par des forts courants tels que la Manche ou le détroit de Behring). Cette valeur est notable si on la compare à l'énergie sauvage dissipée dans les rivières (35000 TWh). Mais, il faut prendre cette comparaison avec la plus grande prudence. L'énergie fournie par une usine hydroélectrique ordinaire était dissipée par frottement dans la rivière quand l'usine n'existait pas. Il y a simplement déplacement du lieu où l'énergie potentielle de l'eau est restituée à l'atmosphère. Le système thermique Soleil-Terre n'est pas modifié.

La proportion de l'énergie "sauvage" pouvant être utilisée est nécessairement inférieure à l'unité, mais seules des conditions pratiques et économiques s'opposent à ce que cette proportion soit importante (5\% actuellement, $10 \%$ peut-être en 2000 ).

L'utilisation de l'énergie des marées pose des problèmes d'une nature toute différente.

En théorie, rien ne s'oppose à produire une énergie supérieure à celle qui était dissipée naturellement, mais en pratique, seule une proportion très faible pourra être utilisée ( 2 à $3 \%)$.

Le tableau I récapitule les principaux sites marémoteurs du monde.

Nous avons essayé d'éviter les erreurs les plus courantes faites dans des récapitulations de ce genre qui rendent les données relatives aux différents sites difficilement comparables.

On les caractérise en général par trois grandeurs : - l'amplitude de la marée (sans préciser nécessairement de quelle amplitude il s'agit : moyenne arithmétique, quadratique, de vives eaux moyennes, etc.) - la surface du bassin (sans préciser à quel niveau elle est prise)

- la longueur de la bouchure qui n'est pas à notre avis un élément caractéristique essentiel d'un projet, car le prix des ouvrages dépend en définitive autant de la profondeur et de la nature des fonds que de la longueur de la digue.

Dans le tableau 1 , nous indiquons:

Pour l'amplitude de la marée : A mètres = la moyenne arithmétique de l'amplitude évaluée sur la période de 18 ans $2 / 3$ - (c'est à quelques pour cent près l'amplitude de l'onde M2). L'expérience nous a montré que pour la plupart des sites, l'amplitude moyenne caractérisait bien le productible.
Pour la surface du Bassin : $S \mathrm{~km}^{2}=$ nous avons indiqué chaque fois que c'était possible la surface au niveau moyen $+A / 4$ qui donne approximativement, dans les cas d'une exploitation à simple effet, la surface du bassin à parois verticales qui fournirait la même production.

Avec les conventions ci-dessus, l'expression $1 / 2 A^{2} S$ donne approximativement en GWh la production annuelle qu'il est possible de tirer d'un site.

Nous avons fait figurer dans le tableau II les aménagements en exploitation ou en cours de réalisation et dans le tableau III ceux ayant fait l'objet d'études récentes.

\section{Quoi de nouveau en 1981-1982 pour l'énergie marémotrice?}

Je retiendrai cinq faits principaux :

1) Achèvement, courant 1981 , du rapport sur l'aménagement de la Severn par le Comité créé à cet effet par le Gouvernement Anglais ;

2) Commande d'un groupe expérimental à alternateur périphérique à installer dans une digue existante sur le site d'Annapolis sur la Baie de Fundy au Canada; 3) Achèvement en fin 1981 par le Groupement SOGREAH-LCHF, appuyé par E.D.F., du projet de l'aménagement de Garolim en Corée du Sud ;

4) Engagement des études de faisabilité du projet de Kutch en Inde ;

5) Reprise par E.D.F. en 1980 de l'étude des possibilités d'utilisation de l'énergie marémotrice sur les côtes de la Manche.

\section{I) Severn (Grande-Bretagne)}

L'estuaire de la Severn sur la côte ouest des lles Britanniques, entre Cornouailles et pays de Galles, constitue un site exceptionnel (amplitude moyenne 8,5 mètres) dont l'utilisation est envisagée de longue date. Les études préliminaires les plus récentes ont été achevées en 1981 et publiées.

Pour nous en tenir à l'essentiel, nous n'évoquerons que le projet "amont" dont les auteurs recommandent la prise en considération en priorité.

Rappelons aussi que l'aménagement de la Severn se heurte à deux difficultés : une activité maritime très importante et une intense mise en suspension des fonds à chaque marée.

Le projet respecte évidemment la première contrainte ( 2 écluses de $336 \mathrm{~m}$ par $50 \mathrm{~m}$ sont prévues).

Des études restent, par contre, à faire pour s'assurer que la modification des mouvements d'eau, à l'aval et à l'amont du barrage, n'entrainera pas de sédimentation ou d'érosion gênantes.

Un autre point mérite d'être signalé : la marée exceptionnelle constatée dans l'estuaire de la Severn est un phénomène local et l'on pouvait craindre qu'il ne soit très perturbé par la présence du barrage et l'exploitation de l'usine.

Les études effectuées à l'aide de plusieurs modèles numériques ont montré en effet, à proximité du bar- 


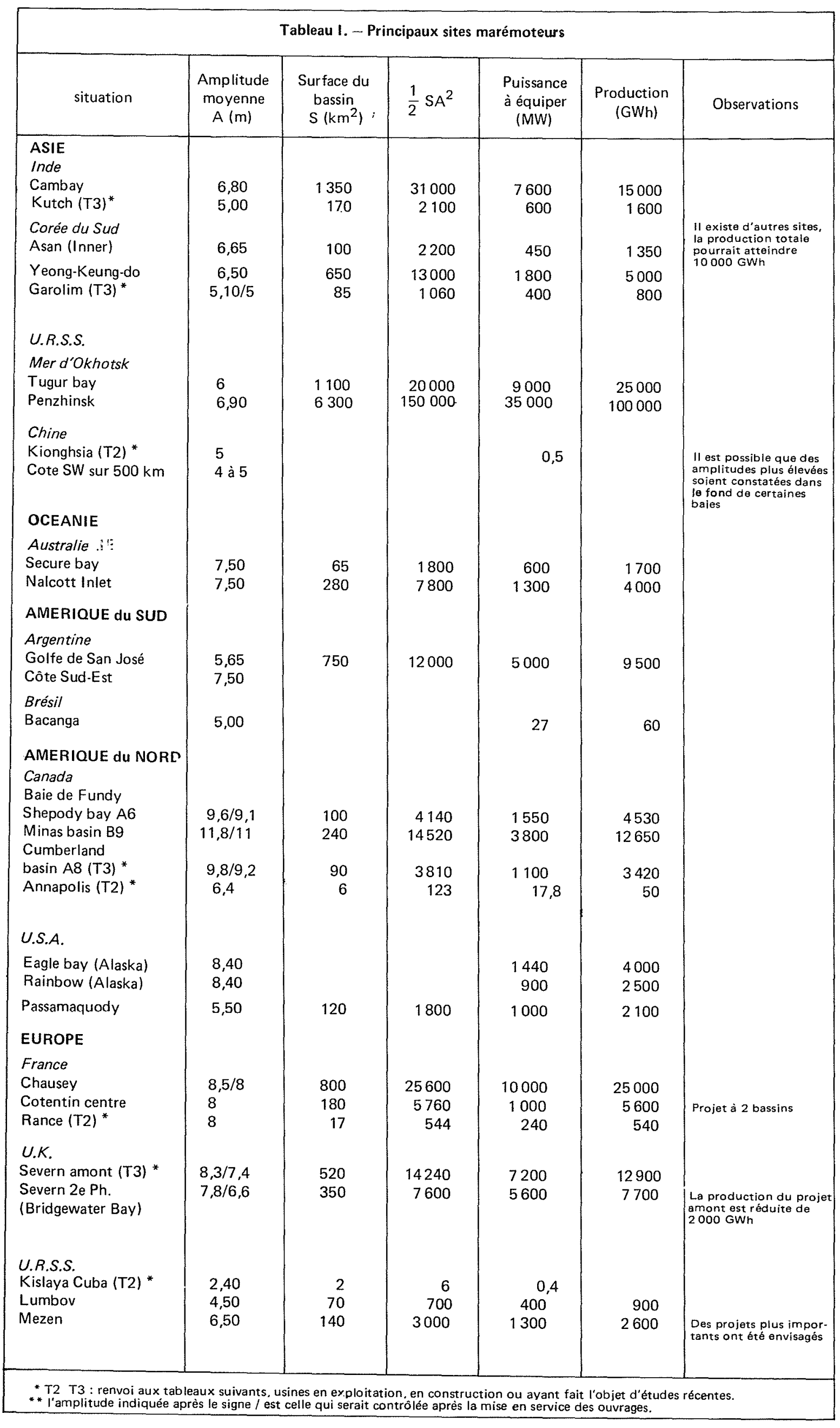




\begin{tabular}{|c|c|c|c|c|c|}
\hline \multicolumn{7}{|c|}{ Tableau II. - Usines marémotrices en service ou en cours de construction } \\
\hline Localisation & $\begin{array}{c}\text { Amplitude } \\
\text { moyenne } \\
(\mathrm{m})\end{array}$ & $\begin{array}{c}\text { Surface du } \\
\text { bassin } \\
\left(\mathrm{km}^{2}\right)\end{array}$ & $\begin{array}{c}\text { Puissance } \\
\text { équipée } \\
\text { (MW) }\end{array}$ & $\begin{array}{c}\text { Productible } \\
\text { annuel } \\
(\mathrm{GWh})\end{array}$ & $\begin{array}{c}\text { Date de mise } \\
\text { en service }\end{array}$ \\
\hline $\begin{array}{c}\text { Rance } \\
\text { (France) }\end{array}$ & 8 & 17 & 240 & 540 & 1966 \\
$\begin{array}{c}\text { Kislaya Cuba } \\
\text { (URSS) }\end{array}$ & 2,4 & 2 & 0,4 & 0,5 & 1968 \\
$\begin{array}{c}\text { Kiansghsia } \\
\text { (Chine) }\end{array}$ & 5 & 6 & 17,8 & 50 & 1985 \\
$\begin{array}{c}\text { Annapolis } \\
\text { (Canada) }\end{array}$ & 6,4 & 6 & & 1980 \\
\hline
\end{tabular}

\begin{tabular}{|c|c|c|c|c|}
\hline \multicolumn{4}{|c|}{ Tableau III. - Projets d'usines marémotrices ayant fait I'objet d'études récentes } \\
\hline \multirow{2}{*}{ Localisations } & $\begin{array}{c}\text { Amplitude } \\
\text { moyenne } \\
(\mathrm{m})\end{array}$ & $\begin{array}{c}\text { Surface du } \\
\text { bassin } \\
\left(\mathrm{km}^{2}\right)\end{array}$ & $\begin{array}{c}\text { Puissance } \\
\text { (INW) }\end{array}$ & $\begin{array}{c}\text { Productible } \\
\text { annuel } \\
\text { (GWh) }\end{array}$ \\
\hline $\begin{array}{c}\text { Severn } \\
\text { (U.K.) }\end{array}$ & $8,3 / 7,4$ & 520 & 7200 & 12900 \\
A6 Canada & $9,6 / 9,1$ & 100 & 1550 & 4530 \\
A8 Canada & $9,8 / 9,2$ & 90 & 1100 & 3420 \\
B9 Canada & $11,8 / 11$ & 240 & 3800 & 12650 \\
$\begin{array}{c}\text { Garolim } \\
\text { (Corée du Sud) }\end{array}$ & 5,10 & 85 & 400 & 800 \\
$\begin{array}{c}\text { Kutch } \\
\text { (Inde) }\end{array}$ & 5,00 & 170 & 600 & 1600 \\
\hline
\end{tabular}

rage, une réduction sensible de la marée dont l'amplitude moyenne passe de $8,3 \mathrm{~m}$ à $7,4 \mathrm{~m}$.

La production de l'usine doit être calculée en conséquence. L'effet s'atténue rapidement vers l'aval.

Le projet retenu est du type : Bassin unique, simple effet à la vidange sans pompage, une "Rance simplifiée" en quelque sorte.

Ses caractéristiques figurent sur le tableau III, les indications qui suivent permettront de se faire une idée plus précise de l'importance de l'ouvrage. Le barrage aurait une longueur totale de $15 \mathrm{~km}$ se répartissant à peu près comme suit :

- Usine : 3,2 km (160 groupes de $45 \mathrm{MW}$ )

- Pertuis de vannes : 4,3 km (22000 $\left.\mathrm{m}^{2}\right)$

- Digues en enrochement : $6 \mathrm{~km}$

- Ecluses et ouvrages de raccordement : 1,5 km

A noter que la plus courte distance des deux points d'ancrage du barrage est de $12 \mathrm{~km}$. Ceci montre bien l'intérêt de choisir un tracé plus en fonction du niveau et de la nature des fonds que pour minimiser la longucur de l'ouvrage.

L'usine serait réalisée en échouant, sur le fond préparé par dragage, des caissons de béton armé. Chaque caisson devant recevoir 3 groupes, aurait une surface à la base de $60 \times 70$ mètres et pèserait environ 100000 tonnes.

Les pertuis à vannes seraient construits sur un principe analogue, les caissons ayant toutefois un moindre encombrement.

Les ouvrages seraient exécutés dans l'ordre suivant : écluses, usine, pertuis, la fermeture de l'estuaire étant effectuée sur des digues en enrochements. La conception des ouvrages et leur mode d'exécution paraissent très analogues dans leur principe à celles qui avaient été recommandées en 1977 pour l'aménagement de la baie de Fundy. 
A noter que les groupes retenus pour la Severn sont plus puissants (diamètre de roue : $9 \mathrm{~m}$ contre $7,5 \mathrm{~m}$ seulement).

Les volumes de matériaux à mettre en cuvre seraient approximativement :

- béton : 4 millions $\mathrm{m}^{3}$;

- sable, enrochement, etc. pour digues : 40 millions $\mathrm{m}^{3}$.

$\mathrm{Ce}$ sont des valeurs qui, repportées à la production annuelle, n'ont rien d'exhorbitant si on les compare à d'autres grands aménagements hydroélectriques.

Les délais d'exécution, bien que très sensiblement réduits par rapport au projet précédent de la Severn, restent importants : 9 ans entre le début des travaux définitifs et la mise en service du premier groupe et 3 ans supplémentaires pour l'achèvement.

\section{2) Groupe d'Annapolis (Canada)}

Il s'agit de créer une véritable usine marémotrice dont les caractéristiques sont données sur le tableau II, qui se placera en deuxième position après l'usine de la Rance.

Le groupe (puissance maximale $20 \mathrm{MW}$, diamètre $7,60 \mathrm{~m}$, alternateur en périphérie de la roue) sera inclus dans un ensemble d'ouvrages existants créés pour contrôler, à des fins agricoles, le niveau dans la rivière Annapolis et en assurer le franchissement par une route : une digue en enrochements de $225 \mathrm{~m}$ de longeur, deux pertuis à vannes et une passe à poissons. L'usine elle-même sera placée dans un îlot compris entre la digue et les vannes.

L'objet essentiel de cette installation est d'essayer un type de groupe qui n'a jusqu'ici été utilisé que pour des usines de rivière et avec des diamètres de roue très inférieurs ( $3 \mathrm{~m} 50$ au maximum).

\section{3) Centrale de Garolim (Corée du Sud)}

L'étude qui vient d'être achevée fait suite à plusieurs études antérieures relatives aux possiblilités d'utilisation de l'énergie marémotrice sur la côte ouest de la Corée.

Bien que l'amplitude de la marée n'atteigne pas des valeurs exceptionnelles, la disposition des lieux ouvre des possibilités très intéressantes. Sur le vue des résultats de ces premières études, les autorités Coréennes ont retenu le site de Garolim. La nouvelle étude a porté uniquement sur ce site dont elle a confirmé les perspectives favorables.

\section{4) Centrale de Kutch (Inde)}

En Janvier 1982 les autorités Indiennes ont donné avec une certaine solennité le coup d'envoi aux études de faisabilité du projet d'usine marémotrice de Kutch à l'extrême Nord-Ouest du territoire Indien (Etat du Gujarat) a proximité immédiate du port de Kandla.

Les caractéristiques principales de ce projet figurent dans les tableaux I et III. Les études nécessitent un important programme de reconnaissances et d'observations qui ont été immédiatement entreprises ${ }^{(3)}$.

(3) La Houille Blanche $n^{\circ} 1,1982$.

\section{5) Etudes françaises}

La France possède sur les côtes de la Manche un des meilleurs sites du monde pour l'utilisation de l'énergie de la marée. Sur plus de $400 \mathrm{~km}$ de côte, l'amplitude moyenne dépasse $6 \mathrm{~m}$, elle dépasse $7 \mathrm{~m}$ sur $300 \mathrm{~km}$ et atteint $8 \mathrm{~m}$ sur une centaine de kilomètres.

La plus grande partie de cette ressource est dans le triangle isocèle rectangle formé par la côte du Cotentin et la côte bretonne, mais l'estuaire de la Somme n'est pas non plus à dédaigner.

En se limitant aux zones de marée moyenne supérieure à 7 mètres, on pourrait produire plus de $50 \mathrm{mil}$ liards de $\mathrm{KWh}$ en doublant presque ainsi le potentiel hydraulique de la France.

Ces conditions naturelles, jointes aux faibles ressources de la France en combustibles fossiles, ont justifié l'intérêt toujours porté par la France à l'énergie des marées; intérêt qui s'est concrétisé dans les années 1950 et 1960 par la réalisation de l'usine de la Rance dans un site particulièrement favorable et l'exécution du programme très étendu d'études et de reconnaissances sur le terrain pour la mise au point d'un projet beaucoup plus vaste. Il s'agissait alors d'une usine à bassin unique et double effet tout à fait analogue dans son principe à celle de la Rance, mais avec des dimensions presque 50 fois plus vastes.

\begin{tabular}{|cc|c|c|c|}
\multicolumn{1}{|c|}{ Tableau IV } \\
\begin{tabular}{|cc|c|c|}
\hline Amplitude \\
moyenne \\
$(\mathrm{m})$
\end{tabular} & $\begin{array}{c}\text { Surface } \\
\text { utile du } \\
\text { bassin } \\
\left(\mathrm{km}^{2}\right)\end{array}$ & $\begin{array}{c}\text { Puissance } \\
\text { equipée } \\
(\mathrm{MW})\end{array}$ & $\begin{array}{c}\text { Production } \\
\text { moyenne } \\
\text { annuelle } \\
(\mathrm{GWh})\end{array}$ \\
\hline $\begin{array}{l}\text { Projet de } \\
\text { Chausey } \\
\text { Usine de } \\
\text { la Rance }\end{array} 8.50 / 8.00$ & 800 & 10000 & 26000 \\
\hline
\end{tabular}

Les études relatives à ce projet ont été mises en sommeil du début des années 1960 jusqu'en 1980, avec toutefois une réévaluation sommaire effectuée en 1975 pour la Commission chargée par le Gouvernement de faire l'inventaire des possibilités d'équipement en énergie hydraulique et marémotrice. Cependant, l'intérêt pour la France d'une utilisation plus large de l'énergie de la marée n'était pas tombé dans l'oubli de tout le monde.

Dans les années 1970, plusieurs Ingénieurs de grande valeur ne manquaient pas une occasion de le rappeler. Citons notamment Albert CAQUOT qui proposait des projets beaucoup plus ambitieux que celui étudié précédemment par E.D.F. et d'une conception différente et Robert GiBRAT. Plusieurs industriels conttinuaient aussi à s'intéresser à cette technique à l'occasion notamment des études faites en dehors de France.

La réouverture du dossier en 1980 par la Direction de l'Equipement a donc suscité immédiatement un grand intérêt dans plusieurs bureaux d'étude qui ont apporté des idées nouvelles sur la manière d'utiliser la ressource d'énergie marémotrice la mieux adaptée à la France. 
Comme nous l'avons indiqué précédemment en effet, tous les projets d'aménagements marémoteurs qui ont été élaborés dans les années rećentes en dehors de France, ont été du type le plus simple : bassin unique, simple effet à la vidange, sans pompage.

Les auteurs de ces projets ont trouvé que c'était ainsi qu'était obtenu le coût moyen du $\mathrm{kWh}$ le plus faible. De tels projets se sont révélés économiquement justifiés si, et seulement si, la plus grande part de leur production se substituait à de l'énergie électrique produite par du pétrole et à la rigueur par du charbon et non à de l'énergie nucléaire.

Or, compte-tenu du programme de centrales nucléaires développé en France, il n'y avait pas de chance pour que cette condition soit réalisée en France même avec l'adjonction du double effet et du pompage. Les nouvelles études se sont donc immédiatement orientées vers les solutions à deux bassins, dont les propriétés - d'ailleurs beaucoup moins bien connues étaient susceptibles de mieux s'adapter aux besoins du réseau français des années futures.

Les cycles à deux bassins décrits au XVIII ${ }^{\mathrm{e}}$ siècle par BELIDOR, mais largement perfectionnés par Albert CAQUOT et ses continuateurs, donnent en effet une beaucoup plus grande liberté dans la programmation $\mathrm{du}$ fonctionnement de l'usine et dans son adaptation aux aléas du réseau. On peut dire, qu'une telle usine était une usine marémotrice plus une usine de pompage ou faire remarquer que la dénivellation maintenue entre les deux bassins permettait à tout instant la fourniture d'une certaine puissance en cas de besoin.

Le coût des ouvrages supplémentaires (digues et vannes) qui en sont la contre-partie, est compensé en partie au moins par une meilleure utilisation de l'usine (3500 heures d'équivalent pleine puissance contre $2500)$ qui se trouve en outre protégée contre la houle.

L'évaluation de la valeur économique de la production est beaucoup plus complexe que pour une usine à simple effet et bassin unique.

Dans ce cas en effet, les irrégularités de la production et des besoins peuvent être considérées comme des variables aléatoires indépendantes.

Avec une usine à deux bassins au contraire, la production s'adaptant aux besoins, sa valeur d'usage ne peut résulter que d'une simulation sur une période assez longue de l'exploitation du réseau et de l'usine en tenant compte des variations et des aléas de l'un et de l'autre.

Les études en cours s'articulent donc autour de trois volets d'importance comparable :

- valeur économique de la production ;

-- coût des ouvrages ;

- effet sur l'environnement.

En parler plus en détail justifierait une nouvelle conférence.

\section{Discussion}

Le Président fait remarquer que l'usine de La Rance est une réalisation technique qui fournit environ ta moitié de l'électricité produite en Bretagne. Les usines marémotrices, source certaine de progrès, sont dans leur version "multibassins" des équipements rentables et certains projets seront certainement réalisés dans les prochaines années.
M. MELER (Escher Wyss, Zurich) présente le groupe Straflo, à alternateur périphérique, conçu pour l'usine marémotrice d'Annapolis au Canada. Il montre ensuite quelques figures concernant cette première installation marémotrice, situé sur la rivière Annapolis qui se jette dans la baie de Fundy, en Nouvelle Ecosse. 


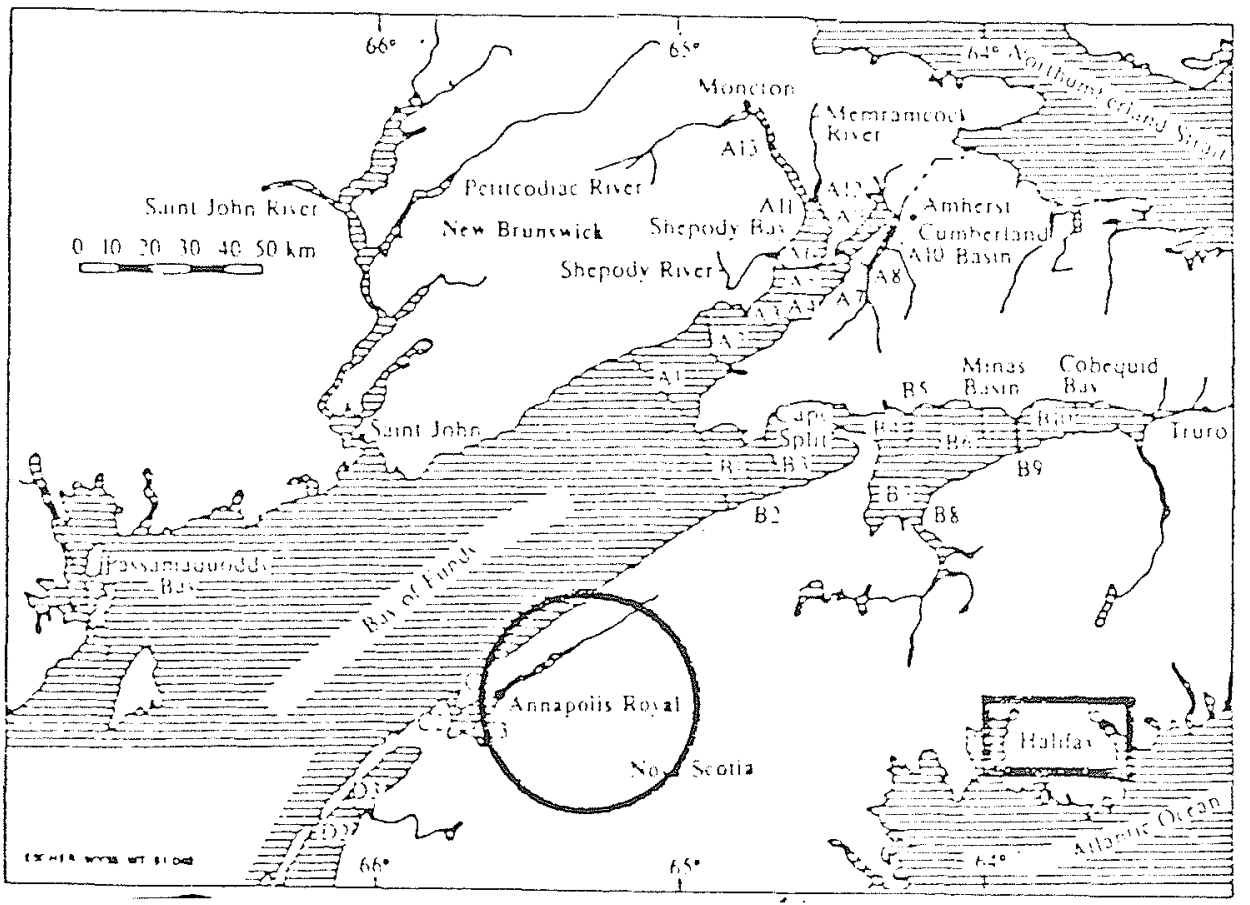

Emplacement de l'usine d'Annapolis en Nouvelle-Ecosse (Canada)

La turbine prévue pour l'usine marémotrice d'Annapolis, dont l'alternateur est disposé à la périphérie de la roue motrice, est la plus puissante de la série des Straflo livrées jusqu'à ce jour. Elle a les caractéristiques suivantes :

Diamètre de la roue motrice . . . . . . . $7,6 \mathrm{~m}$

Nombre de pales motrices . . . . . . . . . 4

Nombre de directrices . . . . . . . . . . . . . 18

Domaine normal de chute ........... 1,4-6,8 m
Chute maximale $\ldots \ldots \ldots \ldots \ldots \ldots \ldots \ldots, \quad 7,1 \mathrm{~m}$

Chute nominale . . . . . . . . . . . . $5,5 \mathrm{~m}$

Puissance sous chute nominale ............. 17,8 MW

Puissance maximale ................. $19,9 \mathrm{MW}$

Débit sous chute nominale . . . . . . . . $378 \mathrm{~m}^{3} / \mathrm{s}$

Vitesse de rotation nominale $\ldots \ldots \ldots \ldots \ldots \ldots, 50 \mathrm{tr} / \mathrm{min}$

Production annuelle ............... $50 \mathrm{GWh}$

\section{Abstract}

Tidal Energy

Apart from two very low power plants, the Rance tidal plant is still, fifteen years after its commissioning, the only operational tidal power station in the World.

Despite certain teething problems - easily explained in the case of such an original development - which necessitated the successive shutdown of all the sets to overhaul the generators, the Rance plant is operating remarkably well.

The concrete and steel structures have proven fully able to resist the onslaught of the marine environment and output is at the level originally envisaged, making due allowance for actual operating conditions.

The year 1981 proves this in particular: the net effective output of $500 \mathrm{GWh}$ (deducting the consumption of pumping and auxiliaries) should be increased by approximately $40 \mathrm{GWh}$ to be compared with the scheduled level of output (exceptional outage of sets to overhaul generators, operating conditions making output advantageous at certain hours of the day, etc.)

From a more general point of view, it is recalled that although the energy dispersed naturally by tide currents in the oceans is of the same order of magnitude as the energy dispersed by rivers (approximately one-third), it is impossible from this comparison to make an estimate of the share of this energy which could be harnessed.

The main tidal sites known are listed in Table I, those which have been recently under study are in Table II, and those in operation or under construction in Table III. The descriptive parameters used are in particular:

- the average inter-annual tide range $A$

- the area of basin $\mathrm{S}$ (in $\mathrm{km}^{2}$ ) at a level exceeding the average sea level by one-fourth of the average tidal range;

- the quantity $1 / 2 \mathrm{SA} 2$ then gives the order of magnitude of the annual production potential of the sile.

Although none of the large tidal plants listed are presently under construction, five recent events of significance for tidal energy must be mentioned:

1) The completion in 1981 of the report on the Severn development by the Committee created for this purpose by the British Government.

2) The order placed for an experimental set with a rim generator to be installed in an existing dyke on the Annapolis site in Fundy Bay (Canada).

3) The completion at end 1981 of the Garolim development project in Korea by the SOGREAH-LCHF group with EDF support.

4) The initiation of feasibility studies for the Kutch project in India.

5) Resumption of the study on the possibility of harnessing tidal energy on the Channel coast by EDF in 1980.

The main characteristics of these developments are given in Tables II and III. 